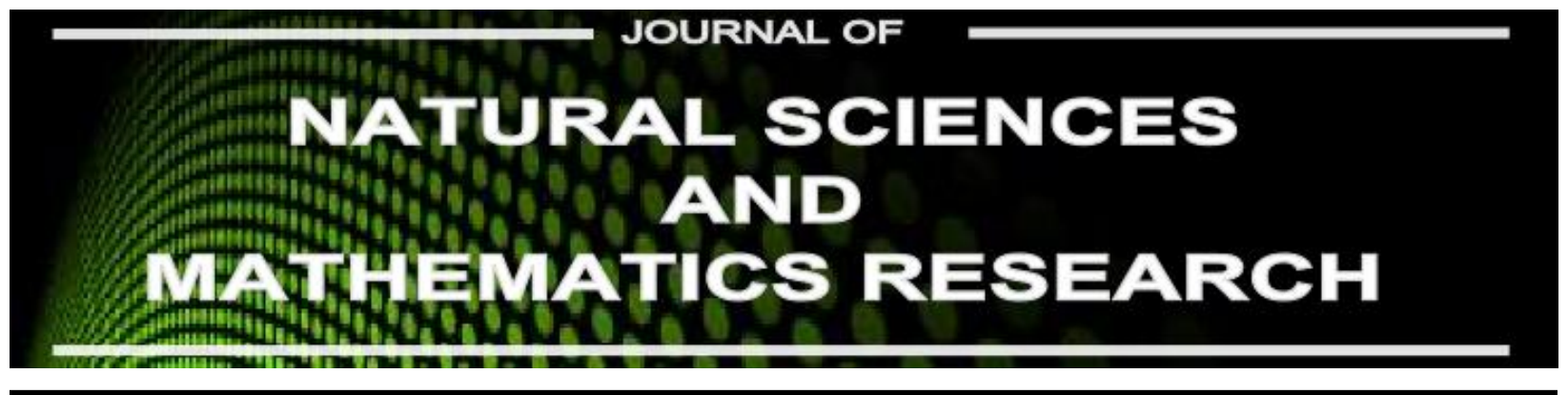

Available online at http://journal.walisongo.ac.id/index.php/jnsmr

\title{
Eigen Value of Fuzzy Matrices
}

\section{Suroto $^{1}$ and Ari Wardayani ${ }^{1}$}

Department of Mathematics, Universitas Jendral Soedirman, Purwokarto, Central Java, Indonesia

\begin{abstract}
s
Corresponding author: Suroto_80@yahoo.com Recived: 20 November 2016, Revised : 20 December 2016 Accepted: 30 December 2016.

In this paper we discuss about eigen value of fuzzy matrices by adopted the substract operation of fuzzy matrices in Sidky and Emam (1992). By applying this substraction, it's yields that some properties eigen value of fuzzy matrices in Joe Anand and Edal Anand is not satisfied.C2016 JNSMR UIN Walisongo. All rights reserved.
\end{abstract}

Keywords: Nilai Eigen; Matriks Fuzzy; Operasi.

\section{Introduction}

The fuzzy semi-ring [1] is a mathematical system formed from the close interval $[0,1]$ and is complemented by addition and multiplication operations i.e.

$$
\begin{aligned}
& a+b=\operatorname{maks}(a, b) \\
& a b=\min (a, b)
\end{aligned}
$$

for each $a, b \in[0,1]$. The fuzzy matrix is a matrix whose entries are elements in the fuzzy semi-ring [2]. In other words a matrix is said to be a fuzzy matrix if $a_{i j} \in[0,1]$. Furthermore, the addition and multiplication operation of this fuzzy matrix is defined as the addition and multiplication operation in the ordinary matrix, but the addition and multiplication operations on the entries adopt from the addition and multiplication operation in the fuzzy sem iring. For example $A=\left[a_{i j}\right]_{m \times n} \quad$ and
$B=\left[b_{i j}\right]_{m \times n}$ Each is a fuzzy matrix then the sum of the matrix $A$ and $B$ are $A+B=\left[a_{i j}+b_{i j}\right]_{m \times n}=\left[\operatorname{maks}\left(a_{i j}, b_{i j}\right)\right]_{m \times n}$

In addition, for example $A=\left[a_{i j}\right]_{m \times n}$ and $B=\left[b_{i j}\right]_{n \times t}$ Each fuzzy matrix, as well $k$ Is scalar in fuzzy semi-ring then multiplication result $A$ and $\mathrm{B}$, as well as scalar multiplication products $k$ with $A$ are,

$$
\begin{gathered}
A B=\left[\sum_{k=1}^{n} a_{i k} b_{k j}\right]_{m \times t}=\left[\operatorname{maks}\left(\min \left(a_{i k}, b_{k j}\right)\right)\right]_{m \times t} \\
k A=\left[k \cdot a_{i j}\right]_{m \times n}=\left[\min \left(k, a_{i j}\right)\right]_{m \times n} .
\end{gathered}
$$

Meanwhile, in Sidky and Emam [3] described the reduction operations in the fuzzy semi-ring, i.e.

$$
a-b= \begin{cases}a, & \text { for } a>b \\ 0, & \text { for } a \leq b_{w}\end{cases}
$$


for each $a, b \in[0,1]$. By adopting a reduction operation in a fuzzy semi-ring, a defined fuzzy matrix reduction operation is defined

$$
A-B=\left[a_{i j}-b_{i j}\right]_{m \times n}
$$

fuzzy every fuzzy matrix $A=\left[a_{i j}\right]_{m \times n}$ and $B=\left[b_{i j}\right]_{m \times n}$. Wang [4] it is explained that a fuzzy matrix is invertible if and only if the matrix is a permutation matrix. Furthermore, Joe Anand and Edal Anand [5] describe some properties related to the eigenvalues of the fuzzy matrix but do not explain the definition of the reduction operation.

This paper will discuss the eigenvalues of a fuzzy matrix on Joe Anand and Edal Anand [5] by adopting the definition of fuzzy matrix reduction as in Sidky and Emam [3]. In the main part of this paper contains the eigenvalues of a fuzzy matrix and some case examples. Furthermore, there will be some related properties of the eigenvalues of a fuzzy matrix with respect to the nature of eigen values in Joe Anand and Edal Anand [5].

\section{Eigen Value of Fuzzy Matrix}

For example a square fuzzy matrix is known $A$, Fuzzy vector and a fuzzy scalar $\alpha$ which satisfies the equation $\alpha X=A X$. Thus the equation $\alpha X=A X$ If and only if $A X-\alpha I X=(A-\alpha I) X=0$, with $I$ is The identity matrix has an order $n$. The system of these equations will be consistent and have a non trivial solution if $|A-\alpha I|=0$. This equation is then called the characteristic equation of the matrix $A$. By solving this equation we get $n$ root fruit for $\alpha$, and the roots are called the characteristic roots or eigenvalues of the matrix $A$ [4].

For each corresponding $\alpha$ value then the equation $\alpha X=A X$ has a nonzero vector solution $X$. For example $X_{t}$ Is a nonzero vector that satisfies the equation $\alpha X=A X$, Then at the moment $\alpha=\alpha_{t}$ then $X_{t}$ is said to be the eigenvector of matrix $A$ corresponding to $\alpha_{t}$. Decomposition results from $|A-\alpha I|$ will produce a polynomial named fuzzy matrix characteristic polynomial $A$ [5].

For example, known fuzzy matrix $A=\left(\begin{array}{ll}0.1 & 0.2 \\ 0.7 & 0.3\end{array}\right) \quad$ Then the characteristic polynomial of the fuzzy matrix $A$ is obtained from $|A-\alpha I|$ i.e

$$
\begin{aligned}
& \left|\left(\begin{array}{ll}
0.1 & 0.2 \\
0.7 & 0.3
\end{array}\right)-\alpha\left(\begin{array}{ll}
1 & 0 \\
0 & 1
\end{array}\right)\right| \\
& \quad=\left|\left(\begin{array}{ll}
0.1 & 0.2 \\
0.7 & 0.3
\end{array}\right)-\left(\begin{array}{cc}
\alpha .1 & \alpha .0 \\
\alpha .0 & \alpha .1
\end{array}\right)\right| \\
& \quad=\left|\left(\begin{array}{ll}
0.1 & 0.2 \\
0.7 & 0.3
\end{array}\right)-\left(\begin{array}{cc}
\min (\alpha, 1) & \min (\alpha, 0) \\
\min (\alpha, 0) & \min (\alpha, 1)
\end{array}\right)\right| \\
& \quad=\left|\left(\begin{array}{ll}
0.1 & 0.2 \\
0.7 & 0.3
\end{array}\right)-\left(\begin{array}{cc}
\alpha & 0 \\
0 & \alpha
\end{array}\right)\right| \\
& =\left|\left(\begin{array}{cc}
0.1-\alpha & 0.2-0 \\
0.7-0 & 0.3-\alpha
\end{array}\right)\right| \\
& =\left|\left(\begin{array}{cc}
0.1-\alpha & 0.2 \\
0.7 & 0.3-\alpha
\end{array}\right)\right| \\
& =(0.1-\alpha)(0.3-\alpha)-(0.2)(0.7) \\
& =\min ((0.1-\alpha),(0.3-\alpha))-\min ((0.2),(0.7)) \\
& =\min ((0.1-\alpha),(0.3-\alpha))-0.2
\end{aligned}
$$

Next to find the characteristic root value of the matrix $A$ i.e

$$
\begin{aligned}
& |A-\alpha I|=0 \\
& \Leftrightarrow \min ((0.1-\alpha),(0.3-\alpha))-0.2=0 \\
& \Leftrightarrow \min ((0.1-\alpha),(0.3-\alpha)) \leq 0.2
\end{aligned}
$$

Case I

For example, $\min ((0.1-\alpha),(0.3-\alpha))=(0.1-\alpha)$ then the results obtained $(0.1-\alpha) \leq 0.2$. possibilities are:

i. $(0.1-\alpha)=0.1$ if $\alpha<0.1$ and comply inequalities $(0.1-\alpha) \leq 0.2$

ii. $(0.1-\alpha)=0$ if $\alpha \geq 0.1$ and comply inequalities $(0.1-\alpha) \leq 0.2$

Thus for each $\alpha \in[0,1]$ will comply inequalities $(0.1-\alpha) \leq 0.2$. 
J. Nat. Scien. \& Math. Res. Vol. 2 No.2 (2016) 181-185, 183

Case II

For exampel,

$\min ((0.1-\alpha),(0.3-\alpha))=(0.3-\alpha)$ the results obtained $(0.3-\alpha) \leq 0.2$. The possibilities are:

i. $(0.3-\alpha)=0.3$ if $\alpha<0.3$ and notcomply inequalities $(0.3-\alpha) \leq 0.2$

ii. $(0.3-\alpha)=0$ if $\alpha \geq 0.3$ and comply inequalities $(0.3-\alpha) \leq 0.2$

Thus for each $\alpha \geq 0.3$ will comply inequalities $(0.3-\alpha) \leq 0.2$.

From the possibility of case I and case II it is obtained that for each $\alpha \in[0,1]$ comply the equation $|A-\alpha I|=0$. Thus there are infinite roots of characteristic equations of the the matrix $A$.

Next will look for fuzzy vectors $X$ Corresponding to an eigenvalue $\alpha$ comply the equation $(A-\alpha I) X=0$ i.e

$\left(\left(\begin{array}{ll}0.1 & 0.2 \\ 0.7 & 0.3\end{array}\right)-\alpha\left(\begin{array}{ll}1 & 0 \\ 0 & 1\end{array}\right)\right)\left(\begin{array}{l}x_{1} \\ x_{2}\end{array}\right)=\left(\begin{array}{l}0 \\ 0\end{array}\right)$

$\Leftrightarrow\left(\left(\begin{array}{ll}0.1 & 0.2 \\ 0.7 & 0.3\end{array}\right)-\left(\begin{array}{ll}\alpha .1 & \alpha .0 \\ \alpha .0 & \alpha .1\end{array}\right)\right)\left(\begin{array}{l}x_{1} \\ x_{2}\end{array}\right)=\left(\begin{array}{l}0 \\ 0\end{array}\right)$

$\Leftrightarrow\left(\left(\begin{array}{ll}0.1 & 0.2 \\ 0.7 & 0.3\end{array}\right)-\left(\begin{array}{ll}\min (\alpha, 1) & \min (\alpha, 0) \\ \min (\alpha, 0) & \min (\alpha, 1)\end{array}\right)\right)\left(\begin{array}{l}x_{1} \\ x_{2}\end{array}\right)=\left(\begin{array}{l}0 \\ 0\end{array}\right)$

$\Leftrightarrow\left(\begin{array}{ll}0.1-\alpha & 0.2-0 \\ 0.7-0 & 0.3-\alpha\end{array}\right)\left(\begin{array}{l}x_{1} \\ x_{2}\end{array}\right)=\left(\begin{array}{l}0 \\ 0\end{array}\right)$

$\Leftrightarrow\left(\begin{array}{cc}0.1-\alpha & 0.2 \\ 0.7 & 0.3-\alpha\end{array}\right)\left(\begin{array}{l}x_{1} \\ x_{2}\end{array}\right)=\left(\begin{array}{l}0 \\ 0\end{array}\right)$

When eigen is taken $\alpha=0.4$ Then obtained

$$
\begin{aligned}
& \left(\begin{array}{cc}
0.1-0.4 & 0.2 \\
0.7 & 0.3-0.4
\end{array}\right)\left(\begin{array}{l}
x_{1} \\
x_{2}
\end{array}\right)=\left(\begin{array}{l}
0 \\
0
\end{array}\right) \\
& \Leftrightarrow\left(\begin{array}{cc}
0 & 0.2 \\
0.7 & 0
\end{array}\right)\left(\begin{array}{l}
x_{1} \\
x_{2}
\end{array}\right)=\left(\begin{array}{l}
0 \\
0
\end{array}\right) \\
& \Leftrightarrow 0 . x_{1}+0.2 . x_{2}=0 \\
& 0.7 . x_{1}+0 . x_{2}=0 \\
& \Leftrightarrow \min \left(0, x_{1}\right)+\min \left(0.2, x_{2}\right)=0 \\
& \min \left(0.7, x_{1}\right)+\min \left(0, x_{2}\right)=0 \\
& \Leftrightarrow 0+\min \left(0.2, x_{2}\right)=0 \\
& \min \left(0.7, x_{1}\right)+0=0 \\
& \Leftrightarrow \operatorname{maks}\left(0, \min \left(0.2, x_{2}\right)\right)=0
\end{aligned}
$$

$$
\begin{gathered}
\operatorname{maks}\left(\min \left(0.7, x_{1}\right), 0\right)=0 \\
\Leftrightarrow x_{2}=0 \text { and } x_{1}=0
\end{gathered}
$$

Thus obtained vector $X=\left(\begin{array}{l}0 \\ 0\end{array}\right)$ which means vector $X$ is a zero vector. Further examples of this case will be used to discuss some properties of the fuzzy matrix eigen value on Joe Anand and Edal Anand [45

\section{The Character Eigen Value of Fuzzy Matrix}

The properties associated with the fuzzy matrix eigenvalues of Joe Anand and Edal Anand (2015).

Lemma 1. The sum of the fuzzy eigen values of a matrix is the sum of the elements on the main diagonal of the fuzzy matrix or the sum of the fuzzy eigenvalues of a matrix equals the trace of the fuzzy matrix

Based on the previous case example, the statement on Lemma 1 is not necessarily applicable. This can be explained as in the case of a fuzzy matrix $A=\left(\begin{array}{ll}0.1 & 0.2 \\ 0.7 & 0.3\end{array}\right)$ which has an eigen value of each value $\alpha \in[0,1]$. The sum of the eigen values of the fuzzy matrix $A=\left(\begin{array}{ll}0.1 & 0.2 \\ 0.7 & 0.3\end{array}\right)$ is $\sum_{i=1}^{\infty} \alpha_{i}=\operatorname{maks}\left(\alpha_{i}\right)=1$. While the result of trace matrix is fuzzy $A=0.1+0.3=\operatorname{maks}(0.1,0.3)=0.3$. Thus the sum of the eigen values of a fuzzy matrix is not the same as the trace of the fuzzy matrix.

Lemma 2. The characteristic root of the triangular fuzzy matrix is only on the diagonal elements of the fuzzy matrix. Or the fuzzy eigenvalues of the triangular matrix are only elements on the main diagonal of the fuzzy matrix.

For example, the fuzzy triangle matrix is known $A=\left(\begin{array}{cc}0.1 & 0.2 \\ 0 & 0.3\end{array}\right)$ hen the characteristic polynomial of the fuzzy matrix $A$ is obtained from $|A-\alpha I|$ i.e 


$$
\begin{aligned}
& \left|\left(\begin{array}{cc}
0.1 & 0.2 \\
0 & 0.3
\end{array}\right)-\alpha\left(\begin{array}{ll}
1 & 0 \\
0 & 1
\end{array}\right)\right| \\
& =\left|\left(\begin{array}{cc}
0.1 & 0.2 \\
0 & 0.3
\end{array}\right)-\left(\begin{array}{cc}
\alpha .1 & \alpha .0 \\
\alpha .0 & \alpha .1
\end{array}\right)\right| \\
& =\left|\left(\begin{array}{cc}
0.1 & 0.2 \\
0 & 0.3
\end{array}\right)-\left(\begin{array}{cc}
\min (\alpha, 1) & \min (\alpha, 0) \\
\min (\alpha, 0) & \min (\alpha, 1)
\end{array}\right)\right| \\
& =\left|\left(\begin{array}{cc}
0.1 & 0.2 \\
0 & 0.3
\end{array}\right)-\left(\begin{array}{ll}
\alpha & 0 \\
0 & \alpha
\end{array}\right)\right| \\
& =\left|\left(\begin{array}{cc}
0.1-\alpha & 0.2-0 \\
0-0 & 0.3-\alpha
\end{array}\right)\right| \\
& =\left|\left(\begin{array}{cc}
0.1-\alpha & 0.2 \\
0 & 0.3-\alpha
\end{array}\right)\right| \\
& =(0.1-\alpha)(0.3-\alpha)-(0)(0.7) \\
& =\min ((0.1-\alpha),(0.3-\alpha))-\min (0,0.7) \\
& =\min ((0.1-\alpha),(0.3-\alpha))-0
\end{aligned}
$$

Next to find the characteristic root value of the matrix $A$ i.e

$$
\begin{aligned}
& |A-\alpha I|=0 \\
& \min ((0.1-\alpha),(0.3-\alpha))-0=0 \\
& \Leftrightarrow \min ((0.1-\alpha),(0.3-\alpha))=0
\end{aligned}
$$

\section{Case I.}

For example

$$
\min ((0.1-\alpha),(0.3-\alpha))=(0.1-\alpha) \text { then }
$$

the results obtained $(0.1-\alpha)=0$. The possibilities are:

i. $(0.1-\alpha)=0.1$ if $\alpha<0.1$ and notcomply equation $(0.1-\alpha)=0$

ii. $(0.1-\alpha)=0$ if $\alpha \geq 0.1$ and comply equation $(0.1-\alpha)=0$

Thus for each $\alpha \in[0.1,1]$ will always satisfy the equation $(0.1-\alpha)=0$.

\section{Case II}

For exampel

$\min ((0.1-\alpha),(0.3-\alpha))=(0.3-\alpha)$ then the results obtained $(0.3-\alpha)=0$. The possibilities are:

i. $(0.3-\alpha)=0.3$ if $\alpha<0.3$ and notcomply equation $(0.3-\alpha)=0$

ii. $(0.3-\alpha)=0$ if $\alpha \geq 0.3$ and comply equation $(0.3-\alpha)=0$
Thus for each $\alpha \geq 0.3$ will satisfy the equation $(0.3-\alpha)=0$

From the possibility of case I and case II it is obtained that for each $\alpha \in[0.1,1]$ comply equation $|A-\alpha I|=0$. Thus there are infinite roots of characteristic equations of the the matrix $A$.

Thus the eigenvalue in the triangular fuzzy matrix is any of the elements $\alpha \in[0.1,1]$ And not necessarily merely the elements on the main diagonal of the fuzzy matrix $A$. So the statement on Lemma 2 is not necessarily applicable.

Lemma 3. If $\alpha$ Is the eigenvalue of the fuzzy matrix $A$ then $1 / \alpha$ with $\alpha \neq 0$ Is the eigenvalue of the fuzzy matrix $A^{-1}$.

According to the discussion of Wang (1984) it is explained that a fuzzy matrix is invertible if and only if the matrix is a permutation matrix. For example, the fuzzy triangle matrix is known $A=\left(\begin{array}{cc}0.1 & 0.2 \\ 0 & 0.3\end{array}\right)$ Then for each $\alpha \in[0.1,1]$ comply equation $|A-\alpha I|=0$. Thus there is an infinite number of nonzero eigenvalues or non-zero characteristic equations of the matrix $A$. Because of the fuzzy matrix $A$ Is not a $\mathrm{F}$ fuzzy matrix permutation then a fuzzy matrix $A^{-1}$ there is no. Furthermore no eigenvalues can be determined for F fuzzy matrix $A^{-1}$. Thus the statement on Lemma 3 is not necessarily applicable.

Lemma 4. Eigen Vector $X$ of fuzzy matrix $A$ Is not unique

Look again at the fuzzy matrix $A=\left(\begin{array}{ll}0.1 & 0.2 \\ 0.7 & 0.3\end{array}\right)$ which has every eigenvalue $\alpha \in[0,1]$ So it has an infinite number of roots of characteristic equations of the matrix $A$. Vector-vector $X$ comply equation $(A-\alpha I) X=0$ for $\alpha=0.4$ is $X=\left(\begin{array}{l}0 \\ 0\end{array}\right)$ which means vector $X$ Is a zero 
vector. Thus for eigenvalues $\alpha=0.4$ only filled by vectors $X=\left(\begin{array}{l}0 \\ 0\end{array}\right)$, So the corresponding eigenvector with $\alpha=0.4$ Is non-existent, so the statement on Lemma 4 is not necessarily applicable.

\section{Conclusion}

The properties of the eigenvalues in the fuzzy matrix have similar properties with the properties of the oxygen values in the ordinary matrix. A few different things lie only in the addition operation and the multiplication operation on a fuzzy matrix defined as the maximum operation and minimum operation. Furthermore, by adopting a fuzzy matrix reduction operation as in Sidky and Emam and applying such a reduction operation to the discussion of Joe Anand and Edal Anand, some properties not applicable.

\section{Acknowledgment}

The authors wish to thank Department of Mathematics, Universitas Jendral Soedirman for supporting this work.

\section{References}

[1] Y. Chongxin, Y. Jie, L. Mai, and Y. Chengde, The Research of Special Fuzzy Matrices. Qiqihar Light-Chemical Engineering Institute, Qiqihar, China, 1984.

[2] Y. Chongxin, and T. Xiang, Fuzzy Singular Matrix. Dept of Basis Qiqihar College of Light Industry, Qiqihar, China, 1984.

[3] F.I. Sidky, and E.G Emam, Some Remarks on Sections of a Fuzzy Matrix. J.K.A.U. Sci. Vol 4. pp 144-155. 1992.

[4] H. Wang, The Fuzzy Non Singular Matrices. Dept of Basis Liaoyang of Petrochemistry China, 1984.

[5] C.M. Joe Anand, and M. Edal Anand, Eigen Values and Eigen Vectors for Fuzzy Matrices. IJERGS. Vol. 3 Issue 1. pp. 878890, 2015. 